\title{
Mental disorders and risk of suicide attempt in prisoners
}

\author{
Louis Favril ${ }^{*}$ \\ Devon Indig² \\ Craig Gear ${ }^{3}$ \\ Kay Wilhelm ${ }^{4,5}$
}

${ }^{1}$ Faculty of Law and Criminology, Ghent University, Belgium

${ }^{2}$ School of Public Health and Community Medicine, University of New South Wales, Australia

${ }^{3}$ CGA Consulting, Sydney, Australia

${ }^{4}$ Discipline of Psychiatry, University of Notre Dame, Australia

${ }^{5}$ School of Psychiatry, University of New South Wales, Australia

"Author for correspondence: Louis Favril (email: louis.favril@ugent.be)

This manuscript has been peer-reviewed and accepted for publication in Social Psychiatry and

Psychiatric Epidemiology, available online at https://doi.org/10.1007/s00127-020-01851-7 


\section{ABSTRACT}

Background: Mental disorders are overrepresented in prisoners, placing them at an increased risk of suicide. Advancing our understanding of how different mental disorders relate to distinct stages of the suicidal process - the transition from ideation to action-would provide valuable information for clinical risk assessment in this high-risk population.

Methods: Data were drawn from a representative sample of 1212 adults (1093 men) incarcerated across 13 New Zealand prisons, accounting for $14 \%$ of the national prison population. Guided by an ideation-to-action framework, three mutually exclusive groups of participants were compared on the presence of mental disorders assessed by validated DSM-IV diagnostic criteria: prisoners without any suicidal history (controls; $n=778$ ), prisoners who thought about suicide but never made a suicide attempt (ideators; $n=187$ ), and prisoners who experienced suicidal ideation and acted on such thoughts (attempters; $n=247$ ).

Results: One-third (34.6\%) of participants reported a lifetime history of suicidal ideation, of whom $55.6 \%$ attempted suicide (19.2\% of all prisoners). Suicidal outcomes in the absence of mental disorders were rare. Whilst each disorder increased the odds of suicidal ideation (OR range 1.734.13) and suicide attempt (OR range 1.82-4.05) in the total sample $(n=1212)$, only a select subset of disorders was associated with suicide attempt among those with suicidal ideation $(n=434)$. Drug dependence $(\mathrm{OR}=1.65,95 \% \mathrm{Cl} 1.10-2.48)$, alcohol dependence $(\mathrm{OR}=1.89,95 \% \mathrm{Cl} 1.26-2.85)$, and posttraumatic stress disorder $(\mathrm{OR}=2.09,95 \% \mathrm{Cl}$ 1.37-3.17) distinguished attempters from ideators. Conclusion: Consistent with many epidemiological studies in the general population, our data suggest that most mental disorders are best conceptualized as risk factors for suicidal ideation rather than for suicide attempt. Once prisoners consider suicide, other biopsychosocial factors beyond the mere presence of mental disorders may account for the progression from thoughts to acts of suicide. Keywords: Psychiatric morbidity, self-harm, suicidal thoughts, ideation-to-action, offenders 


\section{INTRODUCTION}

Suicide is a global public health concern [1] occurring at higher rates in prisoners compared with nonincarcerated people in the community at large [2]. Like many other health outcomes, extant evidence suggests that suicide is rarely the result of a single cause or stressor, but rather depends on the cumulative and interactive effects of myriad biopsychosocial factors [3-7]. Notwithstanding this plethora of risk factors, the presence of a mental disorder is invariably considered one of the most robust and clinically relevant predictors of suicidal behaviour, in the general population [8-16] and in custodial settings alike [17-24]. In their guidelines, many renowned health-oriented and suicidefocused organizations indeed include mental disorders at the top of their lists of suicide risk factors [5]. Accordingly, the early identification and effective management of mental disorders has been widely advocated as a key approach in suicide prevention [25-28], inter alia, by the World Health Organization [29-31].

Although it is well-established that mental disorders are associated with increased risk of suicide, converging evidence highlights that most mental disorders (and many other oft-cited risk factors) actually predict thoughts of suicide (suicidal ideation) rather than suicidal behaviour among those contemplating suicide [32-34]. This thought-behaviour bifurcation is critical, as cross-national data suggest that out of the relatively high proportion of adults who consider suicide, the vast majority (71\%) will not go on to attempt suicide in their lifetime [35]. Similarly, although suicidal ideation conferred an eight-fold increased risk of subsequent suicide attempt in a prospective population-based study, only $7 \%$ of those with baseline suicidal ideation attempted suicide two years later [36]. Therefore, identifying those at greatest risk of acting on their suicidal thoughts can help to improve risk assessment and identify potential targets for intervention in the early phases of the suicidal process - that is, before thoughts progress to acts of suicide. In light of this, large-scale epidemiological research has sought to identify which mental disorders predict suicide attempt beyond their association with suicidal ideation [37-51]. Collectively, results from these methodologically-sound studies generally indicate that, in the few instances where mental disorders 
do predict the transition from ideation to action, these disorders are characterized by anxiety (e.g., posttraumatic stress disorder) and poor impulse control (e.g., substance use disorders). In support of such findings, a meta-analysis of 27 studies recently concluded that only anxiety disorders overall, and posttraumatic stress disorder in particular, as well as drug use disorders, conferred increased risk of suicide attempt among those with suicidal ideation, albeit effect sizes were only small to moderate [52].

In contrast to this accumulating number of studies in the general population, comparatively little is known regarding which mental disorders-and risk factors more generally-uniquely predict the progression from thoughts to acts of suicide among prisoners. Owing to their disproportionally high proportions of both mental disorders [53-56] and suicidal outcomes $[21,57,58]$, communitybased findings should not be generalized to this vulnerable population of incarcerated offenders, or in the very least with caution. To the best of our knowledge, however, only two studies previously investigated clinical factors that differentiate between prisoners who attempted suicide (attempters) and those who thought about suicide but never made an attempt (ideators). In a random sample of 996 adults incarcerated throughout 30 Australian prisons, moderate/severe depression conferred an increased risk of suicide attempt among those who reported suicidal ideation in bivariate analysis, but did not retain statistical significance once other covariates were controlled for [57]. More recently, Favril and O'Connor [58] documented that mental disorders and substance abuse independently distinguished attempters from ideators in a Belgian sample of 1326 prisoners. While yielding important insights, however, these few studies are limited in that they solely relied on a screening instrument for depressive symptomatology [57] or on self-reported diagnoses overall [58], rather than assessing a range of mental disorders through validated diagnostic measures. To date, we are not aware of a single published study examining how specific mental disorders differentially relate to distinct stages of the suicidal process - the transition from ideation to action-among prisoners. Yet, delineating mental disorders that may govern behavioural enaction among those considering suicide could guide risk assessment and enable clinicians to better target interventions, 
especially in this high-risk population where many individuals experience thoughts about suicide [59]. Against this background, we sought to examine the extent to which specific mental disorders are associated with suicide attempt above and beyond their association with suicidal ideation in a nationally representative sample of prisoners.

\section{METHODS}

\section{Participants and procedures}

This paper reports on the 2015 New Zealand Prisoner Mental Health Comorbidity Study which was conducted between March and July 2015 across 13 prisons nationwide. A detailed discussion of the procedures and survey methods can be found in the main study report [60]. Briefly, all prisoners aged 18 years and over who were newly sentenced or in custody for less than three months were invited to participate. Eligible participants were identified by New Zealand Department of Corrections staff and were provided with a brief overview of the study. Those interested in participating were taken to an interview room where members of the National Research Bureau (NRB) informed participants about the study, obtained their signed consent forms, and conducted the face-to-face interviews using computer-assisted personal interviewing. Importantly, the survey was conducted by trained interviewers independent of the prison system (NRB) to ensure objectivity, and study procedures adhered to rigorous protocols to ensure participants' confidentiality and safety. Prisoners were excluded if they did not speak proficient English or if they were determined by correctional staff to be too mentally or physically unwell to participate safely. The average time to complete the interview was 105 minutes, with a median duration of 93 minutes.

Of the 1557 eligible men and women invited to participate in the study, 189 refused to participate and a further 156 were excluded because they did not complete the survey instruments. The final sample of 1212 prisoners (119 women) equates to a $77.8 \%$ response rate, which is similar to a $85 \%$ response found in related prison studies $[57,58]$. The study sample represents $13.8 \%$ of all New Zealand prisoners and was not found to be significantly different by age, sex, or ethnicity [60]. 
Specifically, the sex distribution of the study sample $(90 \%$ men) reflects that of the national prisoner population (96\% men) at the time of recruitment [61].

\section{Measures}

\section{Background variables}

We recorded five demographic characteristics of participants: sex, age $(17-24,25-44$, and $\geq 45$ years), ethnicity (European, Māori, and Pacific Islander or other), country of birth (New Zealand vs. other), marital status (married/de-facto vs. other), and education (secondary school qualification vs. none). Criminological variables included history of incarceration (any prior custody vs. none), custodial status (remand vs. sentenced), security classification (high vs. other), and offence type leading to the current period of imprisonment. This latter variable was recoded into violent (e.g., murder, manslaughter, and rape) vs. non-violent (e.g., drug offences, theft, and fraud) offences.

\section{Mental disorders}

The Composite International Diagnostic Interview (CIDI) 3.0 was used to assess the prevalence of mental disorders [62]. The CIDI is a fully structured and validated diagnostic interview that can be administered by trained lay interviewers, previously adopted in prison settings $[63,64]$. Diagnostic modules were completed for a range of anxiety disorders (generalised anxiety disorder [GAD], panic disorder, and posttraumatic stress disorder [PTSD]), mood disorders (bipolar disorder, dysthymia, and major depressive disorder [MDD]) and substance use disorders (alcohol dependence and drug dependence) which are reported on using DSM-IV diagnoses, as is most commonly used in New Zealand [63]. The survey did not utilise a diagnostic instrument for the assessment of psychotic disorders but used the CIDI questions related to whether a participant indicated that they ever had symptoms of psychosis, such as seeing visions and hearing voices. Personality disorders were assessed using the Personality Diagnostic Questionnaire 4+ (PDQ-4) including the use of the clinical 
significance scale to improve sensitivity and specificity in this prisoner population [65]. All mental disorders were assessed on a lifetime basis.

\section{Suicidal ideation and attempt}

Consistent with similar high-quality studies in prison $[57,58]$ and the general population $[66,67]$, our outcome measure was lifetime history of suicidal ideation and attempt. Suicidal ideation was assessed with the question "Have you ever seriously thought about committing suicide?" (coded as no/yes). Regardless of the answer to the question about suicidal ideation, respondents were also asked whether they ever attempted suicide (coded as no/yes). Both items were used to categorize participants in three mutually exclusive groups: those without any suicidal history (controls), those who had thought about suicide but never made a suicide attempt (ideators), and those who had experienced suicidal ideation and attempted suicide in the past (attempters). No participants were found to have attempted suicide without reporting suicidal ideation.

\section{Statistical analysis}

The final sample was weighted by sex, age, and ethnicity to be a representative sample of the New Zealand prison population as at May 2015. Contingency tables were used to describe characteristics of the total sample, further stratified by participants' suicidal history. A series of logistic regressions were conducted to investigate the relationship of mental disorders with suicide-related outcomes. First, bivariate analyses examined the effects of each disorder separately on suicidal ideation (ideators and attempters vs. controls) and suicide attempt (attempters vs. controls and ideators) in the total sample. For subsequent analyses, the sample was restricted to the subgroup of prisoners who reported suicidal ideation (irrespective of whether they attempted suicide), thus excluding nonsuicidal controls. The rationale behind this choice is that (virtually) all individuals who attempt suicide experience suicidal thoughts, and such a methodological design avoids the problem of accounting for the shared variance with suicidal ideation when examining risk factors for suicide attempt [33]. 
Specifically, we tested whether mental disorders were associated with suicide attempt among those with suicidal ideation (attempters vs. ideators). Both bivariate (i.e., considering only one disorder at a time) and multivariate (i.e., considering all disorders simultaneously) analyses were conducted. The latter (multivariate) analysis sought to identify independent associations between mental disorders and suicide attempt among those with suicidal ideation. We further report on subgroup analyses by participants' sex. Crude (OR) and adjusted (aOR) odds ratios, and their $95 \%$ confidence intervals (Cl), are presented as estimates of the strength of associations, although ORs be overestimated when the prevalence of the outcome is common (>10\%). All analyses were done in SAS (version 9.4), with $p$ values $<0.05$ considered as statistically significant.

\section{RESULTS}

\section{Sample characteristics}

A total of 1212 offenders incarcerated across 13 New Zealand prisons were included in the study. The characteristics of the sample are presented in Table 1, further stratified by their suicidal history (controls, ideators, and attempters). Overall, the majority of participants were men (94.2\%) and born in New Zealand (85.6\%). Their modal age was 25 years (range 17-85), with a mean of 32.6 years (SD $=11.5)$. Three quarters $(75.2 \%)$ of prisoners were sentenced at the time of assessment, with $65.3 \%$ having a history of prior custody. Around one-third (30.7\%) of participants was charged with, or convicted of, a violent offence, and one in five (18.6\%) had a high security classification.

\section{Prevalence estimates}

The weighted lifetime prevalence of suicidal ideation and suicide attempt was $34.6 \%(95 \% \mathrm{Cl} 31.8-$ 37.3) and $19.2 \%$ (95\% Cl 16.9-21.5), respectively. Whereas women were more likely than men to report a suicide attempt ( 28.3 vs. $18.7 ; \mathrm{OR}=1.72,95 \% \mathrm{Cl} 1.13-2.63)$, no sex difference was observed with regard to suicidal ideation ( 42.8 vs. $34.0 ; \mathrm{OR}=1.45,95 \% \mathrm{Cl} 0.98-2.14)$. Of the 1212 participants, 778 (64.2\%) had no suicidal history (controls), 187 (15.4\%) reported suicidal ideation only (ideators), 
and $247(20.4 \%)$ attempted suicide in their lifetime (attempters). All participants who had made a suicide attempt in the past also reported suicidal ideation. Inversely, more than half $(55.6 \%, 95 \% \mathrm{Cl}$ $50.7-60.6)$ of those with suicidal ideation had also attempted suicide (66.1\% of women and $54.8 \%$ of men; $\mathrm{OR}=1.61,95 \% \mathrm{Cl} 0.84-3.07)$.

Table 1. Background characteristics of participants by suicidal history.

\begin{tabular}{|c|c|c|c|c|c|}
\hline & \multirow[b]{2}{*}{$\begin{array}{l}\text { Total sample } \\
\qquad(n=1212)\end{array}$} & \multicolumn{4}{|c|}{ Suicidal history } \\
\hline & & $\begin{array}{l}\text { Controls } \\
(n=778)\end{array}$ & $\begin{array}{l}\text { Ideators } \\
(n=187)\end{array}$ & $\begin{array}{l}\text { Attempters } \\
(n=247)\end{array}$ & $\chi^{2}$ \\
\hline Sex (male) & $1093(94.2)$ & 714 (94.9) & $169(94.5)$ & $210(91.4)$ & $6.30^{*}$ \\
\hline Age group & & & & & 4.05 \\
\hline $17-24$ years & $320(18.9)$ & $214(19.8)$ & $38(13.9)$ & $68(19.8)$ & \\
\hline $25-44$ years & $691(55.7)$ & $436(54.8)$ & $112(57.6)$ & $143(57.2)$ & \\
\hline $45+$ years & $201(25.4)$ & $128(25.4)$ & $37(28.4)$ & $36(23.0)$ & \\
\hline Ethnicity & & & & & $25.63^{*}$ \\
\hline European & $431(33.9)$ & $241(29.8)$ & $85(42.5)$ & $105(40.9)$ & \\
\hline Maori & $623(49.1)$ & $414(49.5)$ & $86(47.4)$ & $123(49.0)$ & \\
\hline Pacific/other & $158(17.0)$ & $123(20.7)$ & $16(10.1)$ & $19(10.1)$ & \\
\hline Country of birth (New Zealand) & 1058 (85.6) & $674(84.0)$ & $163(87.2)$ & $221(89.9)$ & 5.28 \\
\hline Marital status (married) & $606(51.7)$ & $392(52.1)$ & $97(54.2)$ & $117(48.5)$ & 1.35 \\
\hline Secondary school qualification & $463(38.1)$ & $305(39.4)$ & $65(34.3)$ & $93(36.6)$ & 1.81 \\
\hline Any previous custody & $778(65.3)$ & $487(63.7)$ & $128(69.9)$ & $163(67.0)$ & 2.72 \\
\hline Sentenced status & $894(75.2)$ & 589 (76.9) & $136(74.9)$ & $169(69.3)$ & 5.23 \\
\hline High security classification & $222(18.6)$ & $146(19.0)$ & $38(20.7)$ & $38(15.5)$ & 2.00 \\
\hline Violent offence & $384(30.7)$ & 245 (29.9) & $56(30.5)$ & $83(33.5)$ & 1.02 \\
\hline
\end{tabular}

Note. Data are presented as $n(\%)$. All percentages are presented weighted and bases unweighted.

* Significant at the 0.05 level. 
Table 2. Clinical characteristics of participants by suicidal history.

\begin{tabular}{|c|c|c|c|c|c|}
\hline & \multirow[b]{2}{*}{$\begin{array}{l}\text { Total sample } \\
\qquad(n=1212)\end{array}$} & \multicolumn{4}{|c|}{ Suicidal history } \\
\hline & & $\begin{array}{l}\text { Controls } \\
(n=778)\end{array}$ & $\begin{array}{l}\text { Ideators } \\
(n=187)\end{array}$ & $\begin{array}{l}\text { Attempters } \\
\qquad(n=247)\end{array}$ & $\chi^{2}$ \\
\hline \multicolumn{6}{|l|}{ Anxiety disorders } \\
\hline GAD & $108(8.7)$ & $35(4.6)$ & $32(16.5)$ & $41(16.5)$ & $45.81^{*}$ \\
\hline Panic disorder & $65(5.6)$ & $26(3.5)$ & $14(8.1)$ & $25(10.9)$ & $18.10^{*}$ \\
\hline PTSD & 292 (23.9) & $122(15.5)$ & $59(30.3)$ & $111(47.5)$ & $97.31^{*}$ \\
\hline Any anxiety disorder & 372 (29.9) & $158(19.6)$ & $79(41.4)$ & $135(55.7)$ & $116.83 *$ \\
\hline \multicolumn{6}{|l|}{ Mood disorders } \\
\hline Bipolar disorder & $146(11.1)$ & $70(8.1)$ & 32 (15.9) & $44(17.5)$ & $20.57^{*}$ \\
\hline Dysthymia & $60(4.9)$ & $20(2.5)$ & $15(7.9)$ & $25(10.5)$ & $25.64^{*}$ \\
\hline MDD & $254(20.6)$ & $110(14.2)$ & $60(32.5)$ & $84(33.2)$ & $53.18^{*}$ \\
\hline Any mood disorder & $403(32.0)$ & $181(22.4)$ & $93(49.0)$ & $129(51.1)$ & $89.01 *$ \\
\hline \multicolumn{6}{|l|}{ Substance use disorders } \\
\hline Alcohol dependence & 449 (36.3) & $230(29.1)$ & $79(41.1)$ & $140(56.9)$ & $56.81^{*}$ \\
\hline Drug dependence & $479(37.4)$ & $265(31.5)$ & $78(41.6)$ & $136(54.0)$ & $36.71^{*}$ \\
\hline Any substance use disorder & $1058(87.0)$ & $662(84.7)$ & $172(91.3)$ & $224(91.7)$ & $9.68^{*}$ \\
\hline \multicolumn{6}{|l|}{ Other disorders } \\
\hline Any personality disorder & $376(33.1)$ & $207(28.8)$ & $65(37.4)$ & $104(44.3)$ & $18.27^{*}$ \\
\hline Psychosis symptoms & $151(12.6)$ & $50(6.9)$ & $33(18.8)$ & $68(27.0)$ & $64.57^{*}$ \\
\hline Any mental disorder & $1106(90.7)$ & $687(87.7)$ & $181(96.1)$ & $238(96.5)$ & $21.12^{*}$ \\
\hline Two or more disorders & 794 (63.6) & $438(53.8)$ & $146(78.5)$ & $210(85.2)$ & $91.01 *$ \\
\hline
\end{tabular}

Note. Data are presented as $n(\%)$. All percentages are presented weighted and bases unweighted.

GAD = generalised anxiety disorder; PTSD = posttraumatic stress disorder; $\mathrm{MDD}=$ major depressive disorder.

* Significant at the 0.05 level. 
Lifetime prevalence estimates of mental disorders range from a high of $37.4 \%$ for drug dependence to a low of $4.9 \%$ for dysthymia (Table 2). Proportions for any anxiety disorder (29.9\%) and any mood disorder (32.0\%) were comparable, each found in approximately three out of ten prisoners. Any substance use disorder was identified in $87 \%$ of the total sample. According to screening instruments, one-third (33.1\%) of participants had a personality disorder, and $12.6 \%$ reported symptoms of psychosis. In all, nine out of ten (90.7\%) participants met diagnostic criteria for at least one mental disorder in their lifetime, with $63.6 \%$ of the study sample having two or more mental disorders.

\section{Bivariate and multivariate analyses}

Virtually all (96.4\%) participants reporting suicidal ideation met diagnostic criteria for at least one of the disorders assessed, with an identical proportion (96.4\%) found among those who attempted suicide. Among prisoners without any lifetime mental disorder, only 3.6\% reported suicidal ideation and $1.9 \%$ had made a suicide attempt.

The associations of individual mental disorders with suicidal ideation and attempt in the total sample $(n=1212)$ are presented in Table 3 . Bivariate analyses indicate that every single disorder examined was significantly associated with increased odds of suicidal ideation (all $p<0.001$ ), with ORs ranging from 1.73 (personality disorders) to 4.13 (GAD). A similar pattern of results was observed for suicide attempt as the outcome variable, with comparable effect sizes (all $p<0.001$ ). The highest OR for attempted suicide was noted for PTSD $(O R=4.05)$, with the other disorderspecific ORs in the range of 1.82 (personality disorders) to 3.68 (psychosis). Taken together, bivariate ORs for the associations between individual disorders and suicide-related outcomes were all consistently elevated and significant in the total sample of prisoners. As a group, anxiety disorders showed the strongest associations with suicidal ideation $(O R=3.99)$ and attempt $(O R=4.03)$, followed by mood disorders (ORs 3.49 and 2.77) and substance use disorders (ORs 1.95 and 1.81). As a whole, the presence of at least one mental disorder conferred a three-fold increased odds of both 
suicidal ideation $(O R=3.71)$ and suicide attempt $(O R=3.33)$, with slightly higher ORs ( 3.97 and 4.09 respectively) observed among prisoners having two or more disorders.

Although each mental disorder examined was associated with significantly increased odds of suicide attempt in the total sample $(n=1212)$, only three disorders were bivariatly associated with suicide attempt when limiting analyses to prisoners with suicidal ideation $(n=434)$. As detailed in Table 3, drug dependence $(\mathrm{OR}=1.65,95 \% \mathrm{Cl} 1.10-2.48)$, alcohol dependence $(\mathrm{OR}=1.89,95 \% \mathrm{Cl}$ 1.26-2.85), and posttraumatic stress disorder $(\mathrm{OR}=2.09,95 \% \mathrm{Cl} 1.37-3.17)$ conferred a two-fold increase in the odds of suicide attempt among prisoners with suicidal ideation. These ORs represent individual-level associations between specific mental disorders and suicide attempt, without taking into account the possible overlap (comorbidity) of multiple disorders. Therefore, a multivariate regression analysis was further conducted to identify independent associations between all mental disorders and suicide attempt in the subsample of participants reporting suicidal ideation; the results of which are presented in Table $4 .{ }^{1}$ Only PTSD (aOR $\left.=1.17,95 \% \mathrm{Cl} 1.03-1.32\right)$ and alcohol dependence $(\mathrm{aOR}=1.12,95 \% \mathrm{Cl} 1.00-1.26)$ were associated with increased odds of suicide attempt among those reporting suicidal ideation, independently of other disorders. Drug dependence $(\mathrm{aOR}=$ $1.05,95 \% \mathrm{Cl} 0.94-1.19)$ was no longer significant after adjusting for the presence of other mental disorders.

In summary, while mental disorders were consistently associated with suicidal ideation, they had weak (or no) associations with the progression from ideation to attempt.

\footnotetext{
${ }^{1}$ None of the correlations exceeded 0.42 and none of the variance inflation factor (VIF) values were greater than 2 , suggesting that multicollinearity was not an issue in the multivariate model.
} 
Table 3. Bivariate associations between mental disorders and suicidal outcomes.

\begin{tabular}{|c|c|c|c|}
\hline & \multicolumn{2}{|c|}{$\begin{array}{l}\text { In the total sample } \\
\qquad(n=1212)\end{array}$} & \multirow{2}{*}{$\begin{array}{l}\text { Suicide attempt among } \\
\text { those with suicidal } \\
\text { ideation }(n=434)\end{array}$} \\
\hline & Suicidal ideation $^{\mathrm{a}}$ & Suicide attempt & \\
\hline \multicolumn{4}{|l|}{ Anxiety disorders } \\
\hline GAD & $4.13(2.66-6.43)^{*}$ & $2.70(1.75-4.18)^{*}$ & $1.00(0.59-1.71)$ \\
\hline Panic disorder & $2.91(1.72-4.95)^{*}$ & $2.65(1.53-4.58)^{*}$ & $1.38(0.67-2.85)$ \\
\hline PTSD & $3.63(2.71-4.85)^{*}$ & $4.05(2.94-5.58)^{*}$ & $2.09(1.37-3.17)^{*}$ \\
\hline Any anxiety disorder & $3.99(3.04-5.23)^{*}$ & $4.03(2.97-5.48)^{*}$ & $1.78(1.19-2.66)^{*}$ \\
\hline \multicolumn{4}{|l|}{ Mood disorders } \\
\hline Bipolar disorder & $2.28(1.58-3.28)^{*}$ & $2.00(1.34-2.99)^{*}$ & $1.12(0.66-1.91)$ \\
\hline Dysthymia & $3.93(2.19-7.06)^{*}$ & $3.17(1.79-5.60)^{*}$ & $1.36(0.67-2.80)$ \\
\hline MDD & $2.97(2.20-4.02)^{*}$ & $2.32(1.66-3.23)^{*}$ & $1.03(0.67-1.58)$ \\
\hline Any mood disorder & $3.49(2.68-4.55)^{*}$ & $2.77(2.04-3.75)^{*}$ & $1.09(0.73-1.63)$ \\
\hline \multicolumn{4}{|l|}{ Substance use disorders } \\
\hline Alcohol dependence & $2.42(1.87-3.13)^{*}$ & $2.88(2.13-3.91)^{*}$ & $1.89(1.26-2.85)^{*}$ \\
\hline Drug dependence & $2.04(1.58-2.63)^{*}$ & $2.33(1.72-3.16)^{*}$ & $1.65(1.10-2.48)^{*}$ \\
\hline Any substance use disorder & $1.95(1.27-3.00)^{*}$ & $1.81(1.04-3.12)^{*}$ & $1.05(0.50-2.20)$ \\
\hline \multicolumn{4}{|l|}{ Other disorders } \\
\hline Any personality disorder & $1.73(1.32-2.27)^{*}$ & $1.82(1.33-2.48)^{*}$ & $1.33(0.87-2.03)$ \\
\hline Psychosis symptoms & $4.12(2.80-6.07)^{*}$ & $3.68(2.51-5.39)^{*}$ & $1.60(0.97-2.62)$ \\
\hline Any mental disorder & $3.71(2.05-6.73)^{*}$ & $3.33(1.56-7.09)^{*}$ & $1.11(0.37-3.38)$ \\
\hline Two or more disorders & $3.97(2.95-5.35)^{*}$ & $4.09(2.75-6.06)^{*}$ & $1.58(0.93-2.67)$ \\
\hline
\end{tabular}

Note. Data are presented as odds ratios (OR) and their $95 \%$ confidence intervals (CI).

$\mathrm{GAD}=$ generalised anxiety disorder; PTSD = posttraumatic stress disorder; $\mathrm{MDD}=$ major depressive disorder .

* Significant at the 0.05 level.

${ }^{a}$ Irrespective of suicide attempt status. 
Table 4. Multivariate model for suicide attempt among those with suicidal ideation $(n=434)$.

\begin{tabular}{lccc}
\hline & B & SE & aOR $(95 \% \mathrm{Cl})$ \\
\hline GAD & -0.046 & 0.078 & $0.96(0.82-1.11)$ \\
Panic disorder & 0.047 & 0.103 & $1.05(0.86-1.28)$ \\
PTSD & 0.155 & 0.064 & $1.17(1.03-1.32)^{*}$ \\
Bipolar disorder & 0.324 & 0.328 & $1.38(0.73-2.63)$ \\
Dysthymia & 0.014 & 0.108 & $1.01(0.82-1.25)$ \\
MDD & -0.049 & 0.071 & $0.95(0.83-1.09)$ \\
Alcohol dependence & 0.115 & 0.060 & $1.12(1.00-1.26)^{*}$ \\
Drug dependence & 0.052 & 0.060 & $1.05(0.94-1.19)$ \\
Any personality disorder & 0.260 & 0.231 & $1.30(0.82-2.04)$ \\
Psychosis symptoms & 0.233 & 0.258 & $1.26(0.75-2.14)$ \\
\hline
\end{tabular}

Note. $\mathrm{aOR}=$ adjusted odds ratios (adjusted for sex, age, ethnicity, and all other mental disorders in the model) and their $95 \%$ confidence intervals $(\mathrm{Cl})$.

GAD = generalised anxiety disorder; PTSD = posttraumatic stress disorder; MDD = major depressive disorder.

* Significant at the 0.05 level.

\section{Analyses by sex}

In the total sample of men $(n=1093)$, all mental disorders assessed were significantly associated with suicidal ideation (OR range 1.75-3.92) and suicide attempt (OR range 1.85-4.05). Among male prisoners with suicidal ideation $(n=379)$, only PTSD distinguished attempters from ideators, independently of other disorders. Different results were observed among incarcerated women. Only GAD $(O R=9.02)$, PTSD $(O R=4.06)$ and alcohol dependence $(O R=2.72)$ were associated with suicidal ideation in the total female sample $(n=199)$, whereas PTSD (OR $=3.63)$, MDD (OR $=2.79)$, alcohol dependence $(O R=2.42)$ and psychosis symptoms $(O R=14.15)$ were associated with suicide attempt. None of the disorders examined were significantly associated with suicide attempt among female prisoners with suicidal ideation $(n=55)$. We emphasize that the results for female prisoners should be interpreted cautiously given the small sample size and lack of statistical power. 


\section{DISCUSSION}

To date, little is known regarding which mental disorders distinguish prisoners who attempt suicide from those who experience suicidal ideation but do not act on these thoughts. Yet, delineating diagnostic differences between ideators and attempters could shed light on clinical factors that contribute to the translation of suicidal thoughts into behaviour. We sought to addresses this empirical gap, and highlight four key findings from this study involving a representative national sample of more than a thousand New Zealand prisoners.

First, nine out of ten (91\%) prisoners interviewed met diagnostic DSM-IV criteria for at least one mental disorder in their lifetime. Drug and alcohol dependence were the most prevalent disorders, each identified in one-third of participants ( $37 \%$ and $36 \%$ respectively), followed by PTSD and MDD with proportions around $20 \%$ in the study sample. Results further suggest that mental disorders rarely present in isolation; approximately two-thirds (64\%) of participants experienced two or more mental disorders over the lifespan-albeit not necessarily concurrently. These figures are in keeping with those reported in other high-income countries [68-72], underscoring the established finding that mental disorders disproportionately affect the prison population worldwide [53]. Indeed, when compared with findings from a national epidemiological survey of the general New Zealand population [73] and a systematic review of the global prevalence of mental disorders [74], our findings indicate markedly elevated rates for mental disorders among incarcerated offenders.

Second, we estimate that one-third (35\%) of prisoners in New Zealand experienced suicidal ideation in their lifetime, and one-fifth (19\%) had ever attempted suicide. Prior large-scale studies identified highly similar prevalence estimates for suicidal ideation (34-44\%) and suicide attempt (15$22 \%)$ among randomly sampled prisoners $[21,22,57,58]$. In reference to the general population, $16 \%$ and 5\% of 12,992 participants included in the New Zealand Mental Health Survey [75] reported a lifetime history of suicidal ideation and attempt, respectively. Furthermore, among our study participants who reported suicidal ideation, more than half (56\%) had also made a suicide attempt. This ratio of suicide attempts to suicidal ideation is comparable to the $58 \%$ and $47 \%$ respectively 
identified among Australian [57] and Belgian [58] prisoners, but substantially higher than the $29 \%$ found among adults in the wider community [35]. The reason why prisoners appear to be proportionately more susceptible in making the transition from ideation to action is currently unclear and awaits further examination to inform tailored prevention efforts.

Third, we found that suicidal outcomes in the absence of mental disorders were rare [21]. Prisoners meeting diagnostic criteria for at least one mental disorder were more likely to report suicidal ideation and attempt than those with no such disorders. Specifically, every single mental disorder assessed was associated with significantly increased odds of suicidal ideation and attempt in the total sample, supporting the long-held notion that mental disorders are salient predictors of suicidal outcomes in prisoners [17-24]. Importantly, however, these associations were substantially attenuated-and became non-significant in most cases-when examining suicide attempt among prisoners with suicidal ideation. For example, dysthymia conferred a three-fold increased odds of suicide attempt in the total sample, but failed to distinguish attempters from ideators once the shared variance between suicide attempt and suicidal ideation was accounted for. These data suggest that few disorders are associated with suicide attempt above and beyond their association with suicidal ideation. While novel among prisoners, these findings mirror those from more than a dozen large-scale epidemiological studies in non-incarcerated populations [37-51] concluding that most mental disorders are important in the development of suicidal thoughts, but less relevant in predicting which individuals are at greatest risk of acting on these thoughts and progress to a suicide attempt.

Fourth, our results indicate that PTSD and substance use disorders most clearly differentiated between ideators and attempters. Once again, this finding concurs with an established body of literature, suggesting that disorders characterized by increased distress (anxiety) and decreased restraint (poor impulse control) are especially pertinent in predicting the transition from suicidal ideation to action [41-52]. In a similar vein, a recent Belgian study found that substance abuse independently distinguished attempters from ideators in a large prison sample [58]. Despite this 
consistent pattern of findings across populations and settings, however, few empirical studies have yet sought to examine why these specific disorders may govern behavioural enaction among individuals considering suicide. With regard to substance use disorders, a plausible explanation is that the effects of alcohol and/or illicit drug use-either acute or because of long-term sequelae of use-may lower behavioural inhibition and impair decision-making $[76,77]$, making it more likely that one will act upon their suicidal thoughts $[67,78]$. Such deficits in executive functioning have equally been reported in individuals diagnosed with PTSD [79]. Consistent with this assumption, a recent systematic review concluded that attempters are largely similar to ideators on a range of neurocognitive abilities, with the exception of two subdomains of executive functioning: inhibition and decision-making [80]. Pending replication in prison samples, such neurocognitive impairments could potentially explain why certain individuals who are thinking about suicide are propelled towards behavioural enaction, whereas others do not bridge this behavioural threshold [81].

Additionally, recent ideation-to-action theories of suicide [82-85] provide a conceptual framework to understand such differential effects of mental disorders on suicidal ideation vs. attempt. A common tenet shared by these models is an emphasis on the fact that suicidal ideation is only one component of risk and, on its own, is unlikely to result in suicidal behaviour. Although variously defined across theories, each of them posits that both a desire (suicidal ideation) and a capability for suicide are two necessary conditions in order for an individual to attempt suicide. Accordingly, disorders characterized by negative thinking (such as depression) may confer risk by increasing the desire for suicide, whereas those marked by impulsiveness and poor behavioural control (such as substance use disorders) may exacerbate risk by increasing one's capability of acting on suicidal thoughts $[49,86]$. This suicide capability-a necessary component for an individual to progress from ideation to action - is thought to be acquired in a myriad of ways, most notably through exposure to painful and provocative life events [87-89]. By definition, PTSD involves experiencing and reliving such traumatic and potentially life-threatening events, consequently increasing one's capability for suicide. This might explain the well-established finding that PTSD is 
one of the few disorders to consistently predict suicide attempt among those with suicidal ideation [47-52]. Substance use disorders, on the other hand, could equally increase this capability by exposing users to painful and provocative events through proximal (intravenous administration, symptoms of withdrawal, overdose) and distal (engaging in risk-taking and impulsive behaviour while intoxicated) effects resulting from the use of psychoactive substances $[86,90]$. These explanations, however, remain largely hypothetical and in need of further empirical testing in prison samples. Sadly, a decade after Nock and colleagues [49] recommended that future research is warranted to delineate the mechanisms through which people come to think about suicide and subsequently progress from ideation to action, we can only conclude that this proposed line of research is still underdeveloped-yet vital-among this high-risk population of prisoners [58].

\section{Strengths and limitations}

To our knowledge, this paper represents the most comprehensive study in prisoners to date examining the extent to which different mental disorders are associated with suicide attempt beyond their association with suicidal ideation. A noteworthy strength of this study is its large and representative sample, accounting for $14 \%$ of the national prisoner population in New Zealand, all of whom were assessed using validated and reliable diagnostic instruments. Six methodological limitations should nonetheless be borne in mind when interpreting the study findings.

First, the cross-sectional nature of our study prohibits causal inferences and does not afford opportunities to verify the central assumption proposed-that mental disorders occurred temporally prior to suicidal outcomes. Since suicidal history and mental disorders were both assessed on a lifetime basis, the lack of precise temporal ordering may limit our interpretation of the nature of these associations. As such, a number of alternative interpretations cannot be ruled out, including the possibility that PTSD is a direct consequence of having attempted suicide [91] or that substance use reflects a maladaptive coping strategy which emerges after (rather than prior to) a suicide attempt. Second, as no collateral information could be obtained from third-party informants to 
validate participants' reports, data were entirely based on retrospective self-report and thus may be subject to biased recall and social desirability [35,92]. Third, a single-item assessment for both suicidal ideation and attempt was used. Although commonly adopted in similar ideation-to-action studies in prisoner $[57,58]$ and community-based $[66,67]$ populations, such an approach is prone to misclassification [93]. In doing so, we were also unable to tap the intensity or severity of our study outcomes - both the suicide attempt and ideation-only group therefore reflect a heterogeneous composition of suicidal individuals. Future studies should assess characteristics of suicidal thinking (e.g., recency of ideation onset, frequency and uncontrollability of suicidal thoughts, severity of ideation at its worst point, and presence of a suicide plan) as these features have shown to facilitate the transition to suicide attempt $[94,95]$. Fourth, our data examined the presence of non-fatal suicidal outcomes, but not suicide. While suicide attempt history is a robust risk factor of suicide [19], some differences exist in risk factors for fatal and non-fatal suicidal behaviours [96]. Therefore, the current findings may not be generalizable to prisoners who have died by suicide. Fifth, we did not consider the severity or chronicity of each disorder examined, nor did we assess the full range of mental disorders. For example, studies in the general population have shown that obsessivecompulsive disorder [51] and impulse-control disorders (i.e., intermittent explosive disorder, conduct disorder, oppositional defiant disorder, and attention deficit hyperactivity disorder) are associated with suicide attempt among those with ideation [43-50]. These disorders were not included in the study protocol so we were unable to assess their potential relationship to suicidal outcomes. Sixth and finally, we were unable to examine differential associations between mental disorders and suicidal outcomes occurring before or during the current period of incarceration. Prospective studies are warranted to explore whether (and which) mental disorders are longitudinally associated with suicide attempt during the course of imprisonment.

Each of the aforementioned limitations restricts the inferences that can be drawn from this study and represents areas for improvement in future research among this understudied population of incarcerated offenders. 


\section{Conclusion and future directions}

Even within the context of these methodological constraints, our results make a solid contribution to the nascent literature as it advances understanding of the differential associations between mental disorders and distinct stages of the suicidal process among prisoners. A central finding of this study is that the strong relationship observed between most investigated mental disorders and suicide attempt substantively diminished once suicidal ideation was taken into account. Pending replication, our results suggest that only a select subset of disorders-those characterized by anxiety and poor impulse control-may play a role in governing the translation of suicidal thoughts into behaviour.

Despite the fact that prisoners constitute a particularly vulnerable population when it comes to mental health [53-56], our data replicate and extend prior community-based findings showing that most mental disorders may actually predict suicidal ideation, but not the transition from ideation to action [37-51]. Moreover, in the few instances where mental disorders did distinguish attempters from ideators, effect sizes were soberingly small (OR range 1.65-2.09) but nonetheless consistent with recent meta-analytical evidence [52]. Taken together, this recurring pattern of findings across populations and settings suggests that most mental disorders may be important in the development of suicidal ideation, but once individuals consider suicide, other factors beyond the mere presence of mental disorders may account for the progression from thoughts to acts of suicide [32-34,82-85]. This is in keeping with the dominant discourse which emphasizes that suicidal behaviour is a multidetermined phenomenon, and a complex web of synergistically interacting factors is implicated in its aetiology [3-7]. Accordingly, in view of the clinical importance of being able to make predictions about the transition from suicidal ideation to action, further delineating volitional factors that facilitate behavioural enaction among prisoners who consider suicide represents a crucial avenue of future research in this understudied yet high-risk population. 
Author contributions: LF conceived the idea for this paper and designed the methodological plan. DI

had full access to the data and conducted the statistical analyses. LF drafted the manuscript with

input from DI. All authors contributed to the interpretation of the results and subsequent edits of the paper.

Funding: This project was funded by the New Zealand Department of Corrections.

Conflict of interest: All authors declare that the research was conducted in the absence of any commercial or financial relationships that could be construed as a potential conflict of interest.

Ethics statement: Ethical approval was obtained from the Central Health and Disability Ethics

Committee of the Ministry of Health (15/CEN/18).

\section{REFERENCES}

1. Naghavi M (2019) Global, regional, and national burden of suicide mortality 1990 to 2016: systematic analysis for the Global Burden of Disease Study 2016. BMJ 364:194.

2. Fazel $S$, Ramesh $T$, Hawton $K$ (2017) Suicide in prisons: an international study of prevalence and contributory factors. Lancet Psychiatry 4(12):946-952.

3. O'Connor RC, Nock MK (2014) The psychology of suicidal behaviour. Lancet Psychiatry $1(1): 73-85$.

4. Turecki G, Brent DA (2016) Suicide and suicidal behaviour. Lancet 387(10024):1227-1239.

5. Franklin JC, Ribeiro JD, Fox KR, Bentley KH, Kleiman EM, Huang X et al (2017) Risk factors for suicidal thoughts and behaviors: a meta-analysis of 50 years of research. Psychol Bull 143(2):187-232.

6. Turecki G, Brent DA, Gunnell D, O'Connor RC, Oquendo MA, Pirkis J et al (2019) Suicide and suicide risk. Nat Rev Dis Primers 5:74.

7. Fazel S, Runeson B (2020) Suicide. N Engl J Med 382(3):266-274.

8. Chesney E, Goodwin GM, Fazel S (2014) Risks of all-cause and suicide mortality in mental disorders: a meta-review. World Psychiatry 13(2):153-160.

9. Harris EC, Barraclough B (1997) Suicide as an outcome for mental disorders: a meta-analysis. Br J Psychiatry 170(3):205-228.

10. Gili M, Castellví P, Vives M, de la Torre-Luque A, Almenara J, Blasco MJ et al (2019) Mental disorders as risk factors for suicidal behavior in young people: a meta-analysis and systematic review of longitudinal studies. J Affect Disord 245:152-162.

11. Hoertel N, Franco S, Wall MM, Oquendo MA, Kerridge BT, Limosin F et al (2015) Mental disorders and risk of suicide attempt: a national prospective study. Mol Psychiatry 20(6):718726.

12. Too LS, Spittal MJ, Bugeja L, Reifels L, Butterworth P, Pirkis J (2019) The association between mental disorders and suicide: a systematic review and meta-analysis of record linkage studies. J Affect Disord 259:302-313.

13. Knipe D, Williams AJ, Hannam-Swain S, Upton S, Brown K, Bandara P et al (2019) Psychiatric morbidity and suicidal behaviour in low- and middle-income countries: a systematic review and meta-analysis. PLoS Med 16(10):e1002905. 
14. Arsenault-Lapierre G, Kim C, Turecki G (2004) Psychiatric diagnoses in 3275 suicides: a metaanalysis. BMC Psychiatry 4:37.

15. Bertolote JM, Fleischmann A, De Leo D, Wasserman D (2004) Psychiatric diagnoses and suicide: revisiting the evidence. Crisis 25(4):147-155.

16. Cavanagh JTO, Carson AJ, Sharpe M, Lawrie SM (2003) Psychological autopsy studies of suicide: a systematic review. Psychol Med 33(3):395-405.

17. Fazel S, Wolf A, Geddes JR (2013) Suicide in prisoners with bipolar disorder and other psychiatric disorders: a systematic review. Bipolar Disord 15(5):491-495.

18. Baillargeon J, Penn JV, Thomas CR, Temple JR, Baillargeon G, Murray OJ (2009) Psychiatric disorders and suicide in the nation's largest state prison system. J Am Acad Psychiatry Law 37(2):188-193.

19. Fazel S, Cartwright J, Norman-Nott A, Hawton K (2008) Suicide in prisoners: a systematic review of risk factors. J Clin Psychiatry 69(11):1721-1731.

20. Rivlin A, Hawton K, Marzano L, Fazel S (2010) Psychiatric disorders in male prisoners who made near-lethal suicide attempts: case-control study. Br J Psychiatry 197(4):313-319.

21. Jenkins R, Bhugra D, Meltzer H, Singleton N, Bebbington $P$, Brugha T et al (2005) Psychiatric and social aspects of suicidal behaviour in prisons. Psychol Med 35(2):257-269.

22. Sarchiapone M, Carli V, Di Giannantonio M, Roy A (2009) Risk factors for attempting suicide in prisoners. Suicide Life Threat Behav 39(3):343-350.

23. Favril L (2019) Non-suicidal self-injury and co-occurring suicide attempt in male prisoners. Psychiatry Res 276:196-202.

24. Gates ML, Turney A, Ferguson E, Walker V, Staples-Horne M (2017) Associations among substance use, mental health disorders, and self-harm in a prison population: examining group risk for suicide attempt. Int J Environ Res Public Health 14(3):317.

25. Bolton JM, Gunnell D, Turecki G (2015) Suicide risk assessment and intervention in people with mental illness. BMJ 351:h4978.

26. Zalsman G, Hawton K, Wasserman D, van Heeringen K, Arensman E, Sarchiapone $M$ et al (2016) Suicide prevention strategies revisited: 10-year systematic review. Lancet Psychiatry 3(7):646-659.

27. Marzano L, Hawton K, Rivlin A, Smith EN, Piper M, Fazel S (2016) Prevention of suicidal behavior in prisons. Crisis 37(5):323-334.

28. Wasserman D, Rihmer Z, Rujescu D, Sarchiapone M, Sokolowski M, Titelman D et al (2012) The European Psychiatric Association (EPA) guidance on suicide treatment and prevention. Eur Psychiatry 27(2):129-141.

29. WHO (2007) Preventing suicide in jails and prisons. World Health Organization, Geneva.

30. WHO (2014) Preventing suicide: a global imperative. World Health Organization, Geneva.

31. WHO (2013) Mental health action plan 2013-2020. World Health Organization, Geneva.

32. Klonsky ED, May AM (2014) Differentiating suicide attempters from suicide ideators: a critical frontier for suicidology research. Suicide Life Threat Behav 44(1):1-5.

33. Klonsky ED, May AM, Saffer BY (2016) Suicide, suicide attempts, and suicidal ideation. Annu Rev Clin Psychol 12:307-330.

34. Klonsky ED, Qiu TY, Saffer BY (2017) Recent advances in differentiating suicide attempters from suicide ideators. Curr Opin Psychiatry 30(1):15-20.

35. Nock MK, Borges G, Bromet EJ, Alonso J, Angermeyer M, Beautrais A et al (2008) Crossnational prevalence and risk factors for suicidal ideation, plans and attempts. Br J Psychiatry 192(2):98-105.

36. ten Have $M$, de Graaf R, van Dorsselaer S, Verdurmen J, van't Land $H$, Vollebergh $W$ et al (2009) Incidence and course of suicidal ideation and suicide attempts in the general population. Can J Psychiatry 54(12):824-833.

37. Borges G, Angst J, Nock MK, Ruscio AM, Kessler RC (2008) Risk factors for the incidence and persistence of suicide-related outcomes: a 10-year follow-up study using the National Comorbidity Surveys. J Affect Disord 105(1-3):25-33. 
38. Georgiades K, Boylan K, Duncan L, Wang L, Colman I, Rhodes AE et al (2019) Prevalence and correlates of youth suicidal ideation and attempts: evidence from the 2014 Ontario Child Health Study. Canadian Journal of Psychiatry 64(4):265-274.

39. Scocco P, de Girolamo G, Vilagut G, Alonso J (2008) Prevalence of suicide ideation, plans, and attempts and related risk factors in Italy: results from the European Study on the Epidemiology of Mental Disorders-World Mental Health study. Compr Psychiat 49(1):13-21.

40. ten Have M, van Dorsselaer S, de Graaf R (2013) Prevalence and risk factors for first onset of suicidal behaviors in the Netherlands Mental Health Survey and Incidence Study-2. J Affect Disorders 147(1-3):205-211.

41. Kessler RC, Borges G, Walters EE (1999) Prevalence of and risk factors for lifetime suicide attempts in the National Comorbidity Survey. Arch Gen Psychiat 56(7):617-626.

42. Gould MS, King R, Greenwald S, Fisher P, Schwab-Stone M, Kramer R et al (1998) Psychopathology associated with suicidal ideation and attempts among children and adolescents. J Am Acad Child Psy 37(9):915-923.

43. Nock MK, Green JG, Hwang I, McLaughlin KA, Sampson NA, Zaslavsky AM et al (2013) Prevalence, correlates, and treatment of lifetime suicidal behavior among adolescents: results from the National Comorbidity Survey Replication Adolescent Supplement. Jama Psychiat 70(3):300-310.

44. Borges G, Nock MK, Medina-Mora ME, Hwang I, Kessler RC (2010) Psychiatric disorders, comorbidity, and suicidality in Mexico. J Affect Disord 124(1-2):98-107.

45. Borges G, Nock MK, Abad JMH, Hwang I, Sampson NA, Alonso J et al (2010) Twelve-month prevalence of and risk factors for suicide attempts in the World Health Organization World Mental Health Surveys. J Clin Psychiatry 71(12):1617-1628.

46. Millner AJ, Ursano RJ, Hwang I, King A, Naifeh JA, Sampson NA et al (2019) Prior mental disorders and lifetime suicidal behaviors among US Army soldiers in the Army Study to Assess Risk and Resilience in Servicemembers (Army STARRS). Suicide Life Threat Behav 49(1):3-22.

47. Nock MK, Ursano RJ, Heeringa SG, Stein MB, Jain S, Raman R et al (2015) Mental disorders, comorbidity, and pre-enlistment suicidal behavior among new soldiers in the us army: results from the Army Study to Assess Risk and Resilience in Servicemembers (Army STARRS). Suicide Life Threat Behav 45(5):588-599.

48. Naifeh JA, Ursano RJ, Kessler RC, Zaslavsky AM, Nock MK, Dempsey CL et al (2019) Transition to suicide attempt from recent suicide ideation in U.S. Army soldiers: results from the Army Study to Assess Risk and Resilience in Servicemembers (Army STARRS). Depress Anxiety 36(5):412-422.

49. Nock MK, Hwang I, Sampson N, Kessler RC, Angermeyer M, Beautrais A et al (2009) Crossnational analysis of the associations among mental disorders and suicidal behavior: findings from the WHO World Mental Health Surveys. PLoS Med 6(8):e1000123.

50. Nock MK, Hwang I, Sampson NA, Kessler RC (2010) Mental disorders, comorbidity and suicidal behavior: results from the National Comorbidity Survey Replication. Mol Psychiatry 15(8):868-876.

51. Batterham PJ, Calear AL, Christensen H, Carragher N, Sunderland M (2018) Independent effects of mental disorders on suicidal behavior in the community. Suicide Life Threat Behav 48(5):512-521.

52. May AM, Klonsky ED (2016) What distinguishes suicide attempters from suicide ideators? A meta-analysis of potential factors. Clin Psychol 23(1):5-20.

53. Fazel S, Hayes AJ, Bartellas K, Clerici M, Trestman R (2016) Mental health of prisoners: prevalence, adverse outcomes, and interventions. Lancet Psychiatry 3(9):871-881.

54. Baranyi G, Cassidy M, Fazel S, Priebe S, Mundt AP (2018) Prevalence of posttraumatic stress disorder in prisoners. Epidemiol Rev 40(1):134-145.

55. Fazel S, Yoon IA, Hayes AJ (2017) Substance use disorders in prisoners: an updated systematic review and meta-regression analysis in recently incarcerated men and women. Addiction 112(10):1725-1739. 
56. Fazel S, Seewald K (2012) Severe mental illness in 33588 prisoners worldwide: systematic review and meta-regression analysis. Br J Psychiatry 200(5):364-373.

57. Larney S, Topp L, Indig D, O'Driscoll C, Greenberg D (2012) A cross-sectional survey of prevalence and correlates of suicidal ideation and suicide attempts among prisoners in New South Wales, Australia. BMC Public Health 12:14.

58. Favril L, O'Connor RC (2019) Distinguishing prisoners who think about suicide from those who attempt suicide. Psychol Med. https://doi.org/10.1017/S0033291719003118

59. Favril L, Vander Laenen F, Vandeviver C, Audenaert K (2017) Suicidal ideation while incarcerated: prevalence and correlates in a large sample of male prisoners in Flanders, Belgium. Int J Law Psychiatry 55:19-28.

60. Indig D, Gear C, Wilhelm K (2016) Comorbid substance use disorders and mental health disorders among New Zealand prisoners. New Zealand Department of Corrections, Wellington.

61. NZDC (2015) Prison facts and statistics - June 2015. New Zealand Department of Corrections, Wellington.

62. Kessler RC, Ustun TB (2008) The WHO World Mental Health Surveys: global perspectives on the epidemiology of mental disorders. Cambridge University Press, Cambridge.

63. Brinded PMJ, Simpson AIF, Laidlaw TM, Fairley N, Malcolm F (2001) Prevalence of psychiatric disorders in New Zealand prisons: a national study. Aust N Z J Psychiatry 35(2):166-173.

64. Butler T, Indig D, Allnutt S, Mamoon H (2011) Co-occurring mental illness and substance use disorder among Australian prisoners. Drug Alcohol Rev 30(2):188-194.

65. Davison S, Leese M, Taylor PJ (2001) Examination of the screening properties of the Personality Diagnostic Questionnaire 4+ (PDQ-4+) in a prison population. J Pers Disord 15(2):180-194.

66. Wetherall K, Cleare S, Eschle S, Ferguson E, O'Connor DB, O'Carroll RE et al (2018) From ideation to action: differentiating between those who think about suicide and those who attempt suicide in a national study of young adults. J Affect Disord 241:475-483.

67. Mars B, Heron J, Klonsky ED, Moran P, O'Connor RC, Tilling K et al (2019) What distinguishes adolescents with suicidal thoughts from those who have attempted suicide? A populationbased birth cohort study. J Child Psychol Psychiatry 60(1):91-99.

68. Beaudette JN, Stewart LA (2016) National prevalence of mental disorders among incoming Canadian male offenders. Can J Psychiatry 61(10):624-632.

69. Singleton N, Meltzer H, Gateward R (1998) Psychiatric morbidity among prisoners in England and Wales. Office for National Statistics, London.

70. Vicens E, Tort V, Duenas RM, Muro A, Perez-Arnau F, Arroyo JM et al (2011) The prevalence of mental disorders in Spanish prisons. Crim Behav Ment Health 21(5):321-332.

71. Favril L, Dirkzwager A (2019) De psychische gezondheid van gedetineerden in België en Nederland: een systematisch overzicht. Tijdschr Criminol 61(1):5-33.

72. Maccio A, Meloni FR, Sisti D, Rocchi MBL, Petretto DR, Masala C et al (2015) Mental disorders in Italian prisoners: results of the REDiMe study. Psychiatry Res 225(3):522-530.

73. Wells JE, Browne MAO, Scott KM, McGee MA, Baxter J, Kokaua J (2006) Te Rau Hinengaro: the New Zealand Mental Health Survey: overview of methods and findings. Aust N Z J Psychiatry 40(10):835-844.

74. Steel Z, Marnane C, Iranpour C, Chey T, Jackson J, Patel V et al (2014) The global prevalence of common mental disorders: a systematic review and meta-analysis 1980-2013. Int J Epidemiol 43(2):476-493.

75. Beautrais AL, Wells JE, McGee MA, Browne MAO (2006) Suicidal behaviour in Te Rau Hinengaro: the New Zealand Mental Health Survey. Aust N Z J Psychiatry 40(10):896-904.

76. de Wit $\mathrm{H}$ (2009) Impulsivity as a determinant and consequence of drug use: a review of underlying processes. Addict Biol 14(1):22-31. 
77. Kozak K, Lucatch AM, Lowe DJ, Balodis IM, MacKillop J, George TP (2019) The neurobiology of impulsivity and substance use disorders: implications for treatment. Ann N Y Acad Sci 1451(1):71-91.

78. Borges G, Loera CR (2010) Alcohol and drug use in suicidal behaviour. Curr Opin Psychiatry 23(3):195-204.

79. Polak AR, Witteveen AB, Reitsma JB, Olff M (2012) The role of executive function in posttraumatic stress disorder: a systematic review. J Affect Disord 141(1):11-21.

80. Saffer BY, Klonsky ED (2018) Do neurocognitive abilities distinguish suicide attempters from suicide ideators? A systematic review of an emerging research area. Clin Psychol 25(1):e12227.

81. Bredemeier K, Miller IW (2015) Executive function and suicidality: a systematic qualitative review. Clin Psychol Rev 40:170-183.

82. Van Orden KA, Witte TK, Cukrowicz KC, Braithwaite SR, Selby EA, Joiner TE (2010) The interpersonal theory of suicide. Psychol Rev 117(2):575-600.

83. O'Connor RC, Kirtley OJ (2018) The integrated motivational-volitional model of suicidal behaviour. Philos Trans R Soc Lond B Biol Sci 373(1754):20170268.

84. Klonsky ED, May AM (2015) The three-step theory (3ST): a new theory of suicide rooted in the "ideation-to-action" framework. Int J Cogn Ther 8(2):114-129.

85. Klonsky ED, Saffer BY, Bryan CJ (2018) Ideation-to-action theories of suicide: a conceptual and empirical update. Curr Opin Psychol 22:38-43.

86. Silva C, Ribeiro JD, Joiner TE (2015) Mental disorders and thwarted belongingness, perceived burdensomeness, and acquired capability for suicide. Psychiatry Res 226(1):316-327.

87. May AM, Victor SE (2018) From ideation to action: recent advances in understanding suicide capability. Curr Opin Psychol 22:1-6.

88. Smith PN, Cukrowicz KC (2010) Capable of suicide: a functional model of the acquired capability component of the interpersonal-psychological theory of suicide. Suicide Life Threat Behav 40(3):266-275.

89. Smith PN, Selwyn C, D'Amato D, Granato S, Kuhlman S, Mandracchia JT (2016) Life experiences and the acquired capability for suicide in incarcerated men. Death Stud 40(7):432-439.

90. Cheek SM, Nestor BA, Liu RT (2016) Substance use and suicidality: specificity of substance use by injection to suicide attempts in a nationally representative sample of adults with major depression. Depress Anxiety 33(6):541-548.

91. Stanley IH, Boffa JW, Joiner TE (2019) PTSD from a suicide attempt: phenomenological and diagnostic considerations. Psychiatry 82(1):57-71.

92. Simon GE, Vonkorff M (1995) Recall of psychiatric history in cross-sectional surveys: implications for epidemiologic research. Epidemiol Rev 17(1):221-227.

93. Millner AJ, Lee MD, Nock MK (2015) Single-item measurement of suicidal behaviors: validity and consequences of misclassification. PLoS One 10(10):e0141606.

94. Nock MK, Millner AJ, Joiner TE, Gutierrez PM, Han G, Hwang I et al (2018) Risk factors for the transition from suicide ideation to suicide attempt: results from the Army Study to Assess Risk and Resilience in Servicemembers (Army STARRS). J Abnorm Psychol 127(2):139-149.

95. Miranda R, Ortin A, Scott M, Shaffer D (2014) Characteristics of suicidal ideation that predict the transition to future suicide attempts in adolescents. J Child Psychol Psychiatry 55(11):1288-1296.

96. Boren EA, Folk JB, Loya JM, Tangney JP, Barboza SE, Wilson JS (2018) The suicidal inmate: a comparison of inmates who attempt versus complete suicide. Suicide Life Threat Behav 48(5):570-579. 